\title{
Sample Manipulation System for Sample Analysis at Mars
}

\author{
Erik Mumm, Tom Kennedy ${ }^{*}$, Lee Carlson ${ }^{*}$ and Dustyn Roberts ${ }^{*}$
}

\begin{abstract}
The Sample Analysis at Mars (SAM) instrument will analyze Martian samples collected by the Mars Science Laboratory Rover with a suite of spectrometers. This paper discusses the driving requirements, design, and lessons learned in the development of the Sample Manipulation System (SMS) within SAM. The SMS stores and manipulates 74 sample cups to be used for solid sample pyrolysis experiments. Focus is given to the unique mechanism architecture developed to deliver a high packing density of sample cups in a reliable, fault tolerant manner while minimizing system mass and control complexity. Lessons learned are presented on contamination control, launch restraint mechanisms for fragile sample cups, and mechanism test data.
\end{abstract}

\section{Introduction}

The Mars Science Laboratory (MSL) currently scheduled for a Fall 2009 launch is NASA's next roving mission to Mars. The rover is equipped with several instruments designed to assess the habitability of Mars, both past and present. One of the instruments, Sample Analysis at Mars (SAM) provided by Goddard Space Flight Center consists of a Gas Chromatograph Mass Spectrometer, Quadropole Mass Spectrometer, and Tunable Laser Spectrometer. Both atmospheric and solid samples can be analyzed by SAM. SAM's Sample Manipulation System (SMS) stores and manipulates sample cups used for experiments involving solid samples.

The SMS is a first generation flight system. The SMS is responsible for controlled manipulation of the sample from SAM's sample inlet device to pyrolysis ovens, where samples are pyrolyzed and evolved gasses plumbed to the analytical suite. Seventy-four sample cups are populated in two polar arrays on the SMS carousel. Cups may be re-used in an extended mission scenario. Once inserted into the pyrolysis oven, the SMS applies a predetermined force to create a hermetic seal between the sample cup and the interior of the oven. The sensitivity of SAM's spectrometers require the SMS to be very clean so as not to contaminate Martian samples and produce false positives or mask sample signatures with known outgassers within the SMS.

This paper covers the design, test, and preliminary lessons learned during the development of the SMS. Concentration is centered on the overall design of the SMS that includes several mechanisms. Lessons learned from contamination control, and results from environmental testing are presented.

\section{SMS Design}

\section{Driving Requirements}

The purpose of the SMS is to store and manipulate sample cups used to analyze solid samples with SAM's instrument suite. The SMS, Figure 1, accepts solid sample from the MSL rover via SAM's Solid Sample Inlet Device into any of 74 sample cups and transports the selected sample cup to a pyrolysis oven. Once the SMS inserts the sample cup into the oven, it creates a seal force sufficient to provide a leak rate between the cup and the oven less than $1 \mathrm{E}-5 \mathrm{cc}$ He/sec. The SMS must consume less than 6Watts continuous and 18-Watts peak power.

The sample cups are separated into three categories: solid sample quartz cups (60), foil topped metal cups for wet chemistry experiments (10), and foil topped metal cups containing calibration sample (4). The SMS must position each cup within $0.71 \mathrm{~mm}$ true position at multiple interfaces including the solid

\footnotetext{
${ }^{*}$ Honeybee Robotics Spacecraft Mechanisms Corporation, New York, NY

Proceedings of the $39^{\text {th }}$ Aerospace Mechanisms Symposium, NASA Marshall Space Flight Center, May7-9, 2008.
} 
sample inlet device, foil puncture stations for the metal cups, and a pyrolysis oven on each row of the SMS Sample Carousel Disk.

To allow SAM to re-use sample cups in an extended mission scenario, the SMS must deliver up to 1350 Newtons with an accuracy of $\pm 10 \%$ of the commanded seal force. A seal is created with the Pyrolysis Ovens via an annealed copper disk brazed to the sample cup and a titanium knife-edge on the oven. Each successive use of a sample cup requires a larger seal force.

Contamination control played a significant role in SMS development. The spectrometers aboard the SAM suite have the capability to detect molecules in the parts-per-billion range. Thus, the SMS must be extremely clean, free of particles, and exhibit a very low outgassing rate. The outgassing rate of the SMS was required to be less than $4.2 \mathrm{E}-14 \mathrm{~g} / \mathrm{cm}^{2} / \mathrm{sec}$ as measured by a Quartz Crystal Microbalance. This ultimately corresponded to a delta/delta frequency reading on a TQCM of $1 \mathrm{~Hz} /$ hour/hour during bake-out.

The SMS must move the sample from the inlet location and seal it in the pyrolysis oven in five minutes or less. This requirement is driven by the extreme contamination sensitivity. A given sample cup, once preconditioned in the pyrolysis oven, must not be exposed to the interior of the SMS for an extended time to prevent contaminating the measurement on the solid sample. Contamination concerns required the SMS to provide a vacuum seal to prevent contamination of the interior of the SMS during Assembly, Test, and Launch operations.

The SMS must be capable of recovering from an unexpected power loss. Feedback devices and a robust high level control architecture combined to provide full fault detection and correction capability.

\section{High Level Architecture}

The four main subsystems of the SMS are identified in Figure 1. Not shown is a contamination cover that fully encases the SMS within SAM. The SMS is not exposed to the exterior of SAM or the Rover, except when the Sample Inlet Tube $(\sim \varnothing 4.3 \mathrm{~mm})$ is open to deliver a sample.

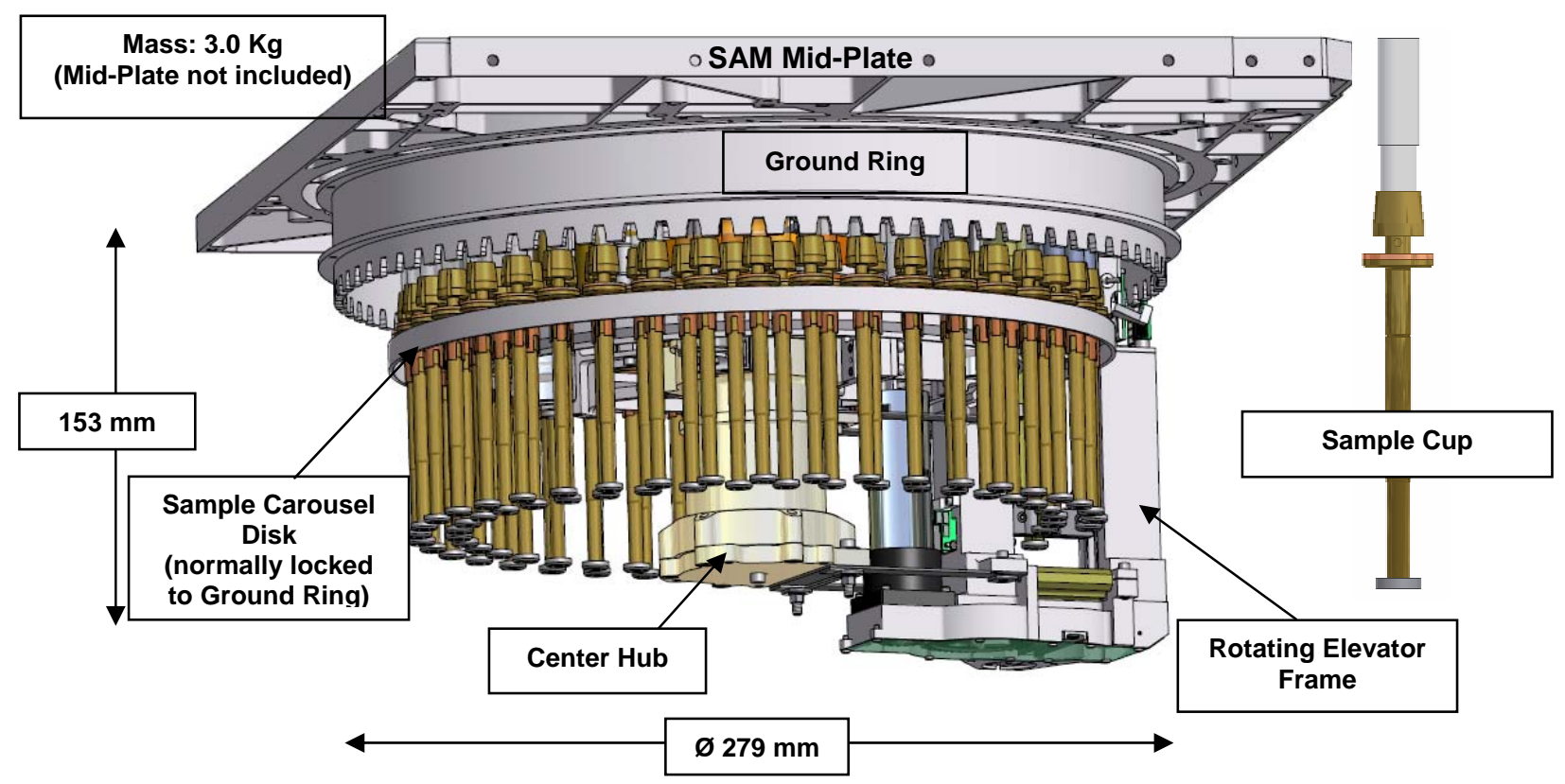

Figure 1 - SMS Main Subsystems

The SMS is an under-actuated 3 Degree-Of-Freedom robotic system. Two rotational degrees of freedom are provided by the Center Hub Actuator. A toggle mechanism on the Sample Carousel Disk either couples the Sample Carousel Disk to the Ground Ring or the Rotating Elevator Frame (REF). When the 
Sample Carousel Disk is coupled to the REF, the Center Hub Actuator positions a given sample cup at an oven, inlet, or puncture station. When the Sample Carousel Disk is coupled to the Ground Ring, the REF rotates independently of the Sample Carousel Disk and positions the Elevator subassembly beneath a given sample cup. At that point, the Elevator actuator ( $3^{\text {rd }}$ DOF) can raise and lower the sample cup to its interface. Figure 2 through Figure 4 demonstrate the sequence of operations involved in decoupling the Sample Carousel Disk from the Ground Ring and moving a cup via the Carousel actuator to another position.

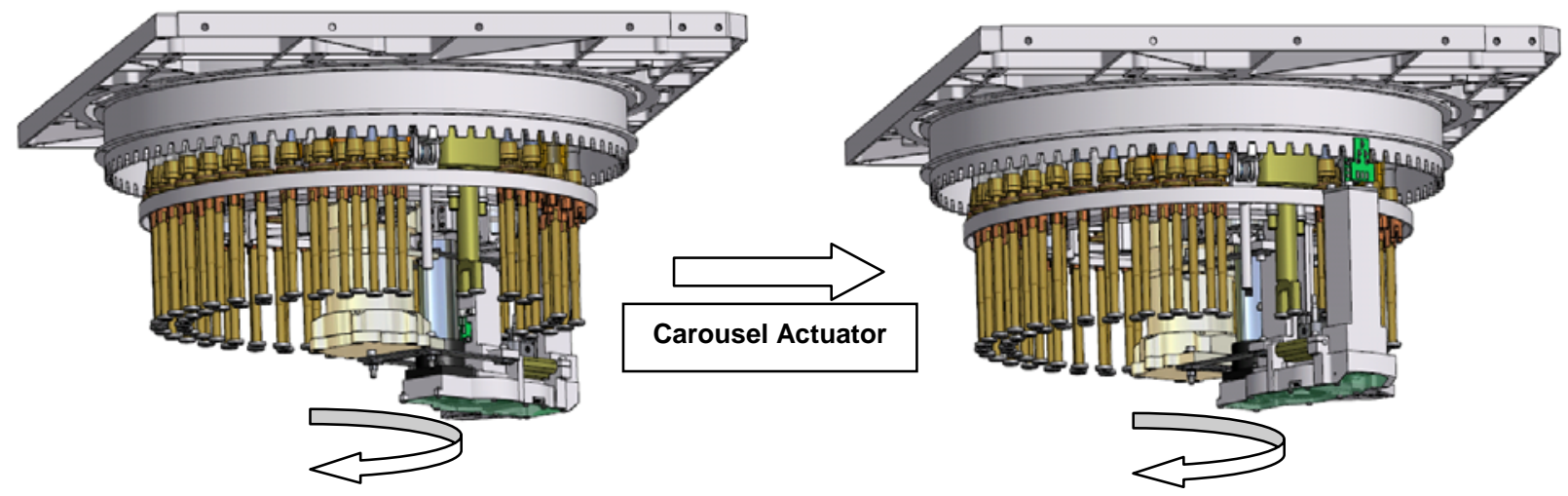

Figure 2 - Center Hub Actuator Moves Rotating Elevator Frame to Toggle Mechanism, Sample Carousel Disk is Stationary

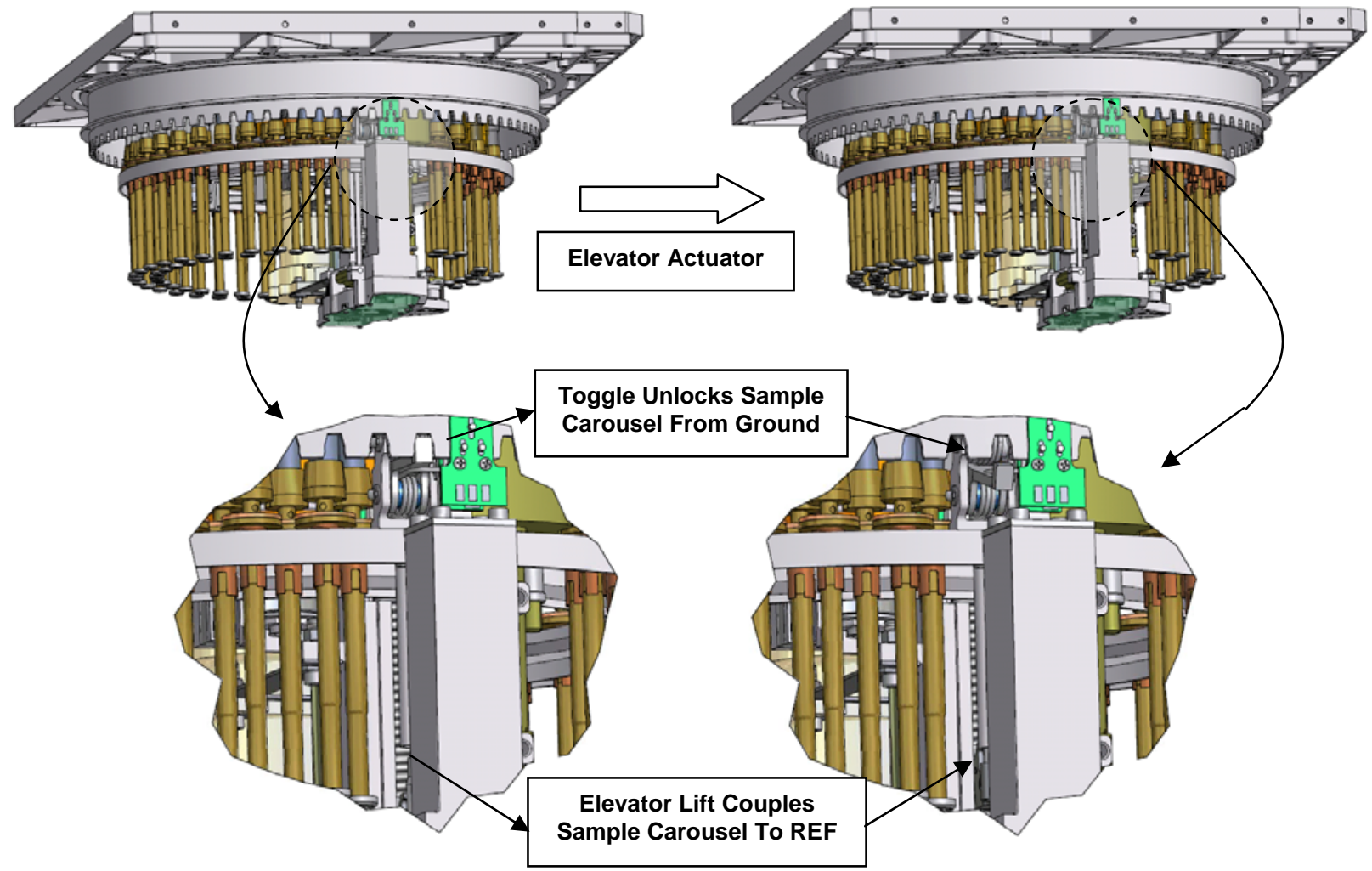

Figure 3 - Elevator Actuator Raises Lift to Unlock Toggle Mechanism from Ground Ring and Couple with Rotating Elevator Frame 


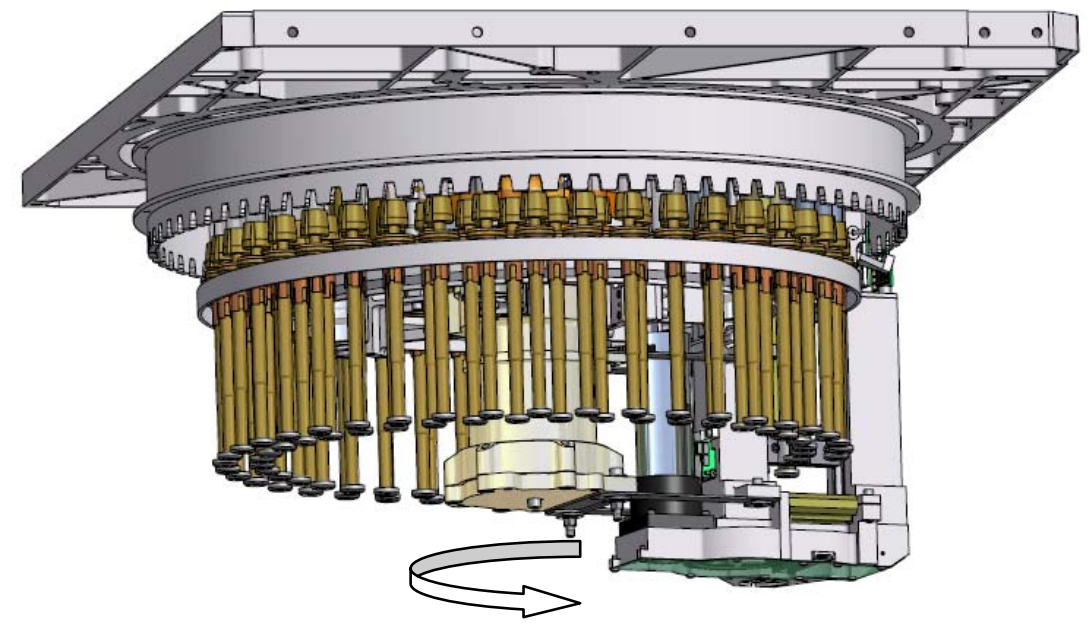

Figure 4 - Center Hub Actuator Moves Rotating Elevator Frame and Sample Carousel Disk to Position a Sample Cup

\section{Center Hub Actuator}

The center hub actuator is comprised of a hall commutated brushless dc motor, 25:1 planetary gearbox, and 100:1 cup style harmonic drive for an overall gear reduction of 2500:1 and 30000 counts per revolution, Figure 5. All components are grease-plated with Braycote 600 EF Micronic. The maximum speed at the output of the harmonic drive is 2 RPM. The maximum continuous torque output is $3.4 \mathrm{~N}-\mathrm{m}$. The Carousel Motor is current limited by the motor control board so as not to exceed output torque limits. Actual running torque of the Center Hub Actuator is only $\sim .05 \mathrm{~N}-\mathrm{m}$ at the output of the $25: 1$ planetary stage. External loads are not significant during operation.

A duplexed pair of angular bearings in the face-to-face configuration is located near the harmonic drive output. A Conrad bearing is located at the interface of the cross-support arms to the center hub. The Center Hub bearings support approximately $70 \%$ of the system mass during launch and EDL, including the Sample Carousel Disk.

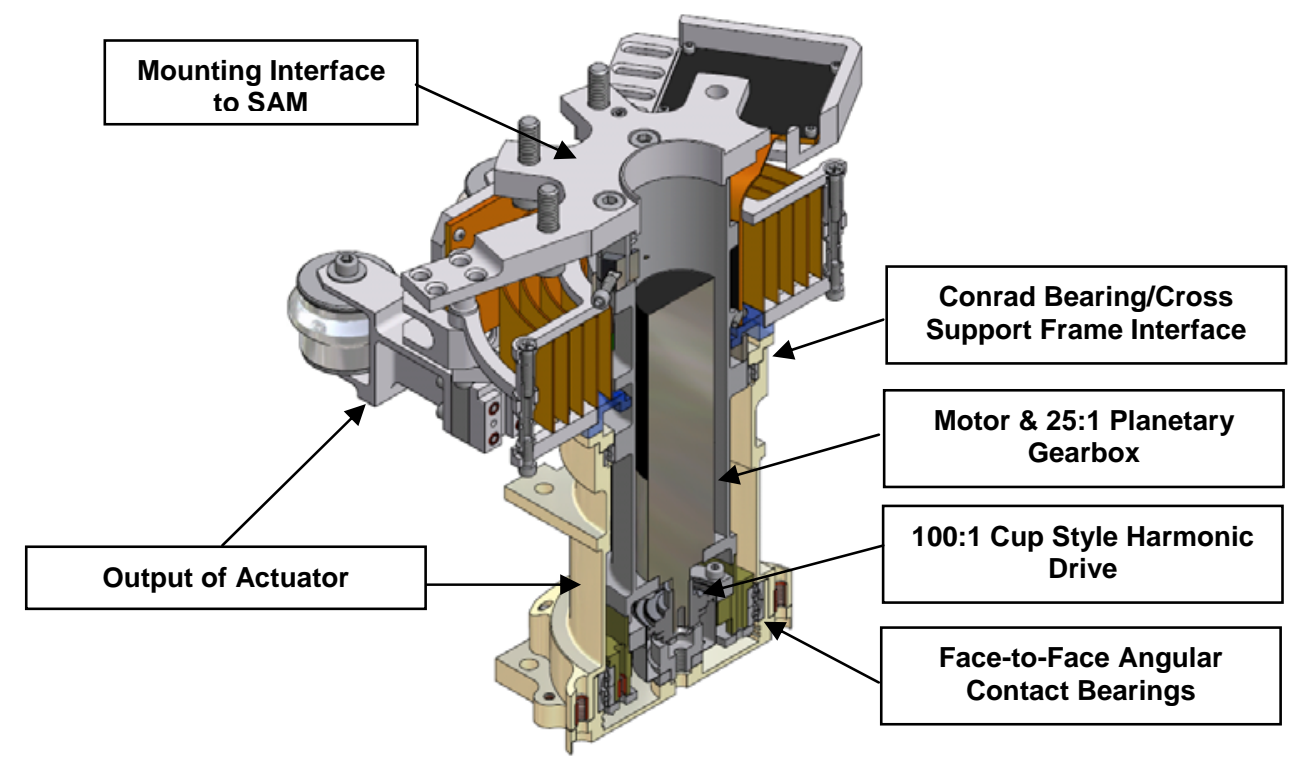

Figure 5 - Center Hub Actuator \& Center Hub Assembly 
The cross support arms support the Sample Carousel Disk via a set of 4 wheels rolling in a v-groove in the Sample Carousel Disk. Each Cross Support Arm wheel contains a set of preloaded angular pair bearings in the back-to-back configuration. The Cross Support Arm wheels roll in the Sample Carousel Disk groove when the Sample Carousel Disk is locked to the Ground Ring, Figure 3.

Due to the tight positioning requirements of the Sample Cups, the Sample Carousel Disk was required to be concentric with respect to the center hub axis of rotation. To accomplish this in the presence of significant manufacturing tolerance stack-up, the radial position of the cross support rollers was adjusted during assembly to ensure the Sample Carousel Disk was concentric to within $0.05 \mathrm{~mm}$. Shims were independently precision ground to desired thicknesses at the assembly level, Figure 6.

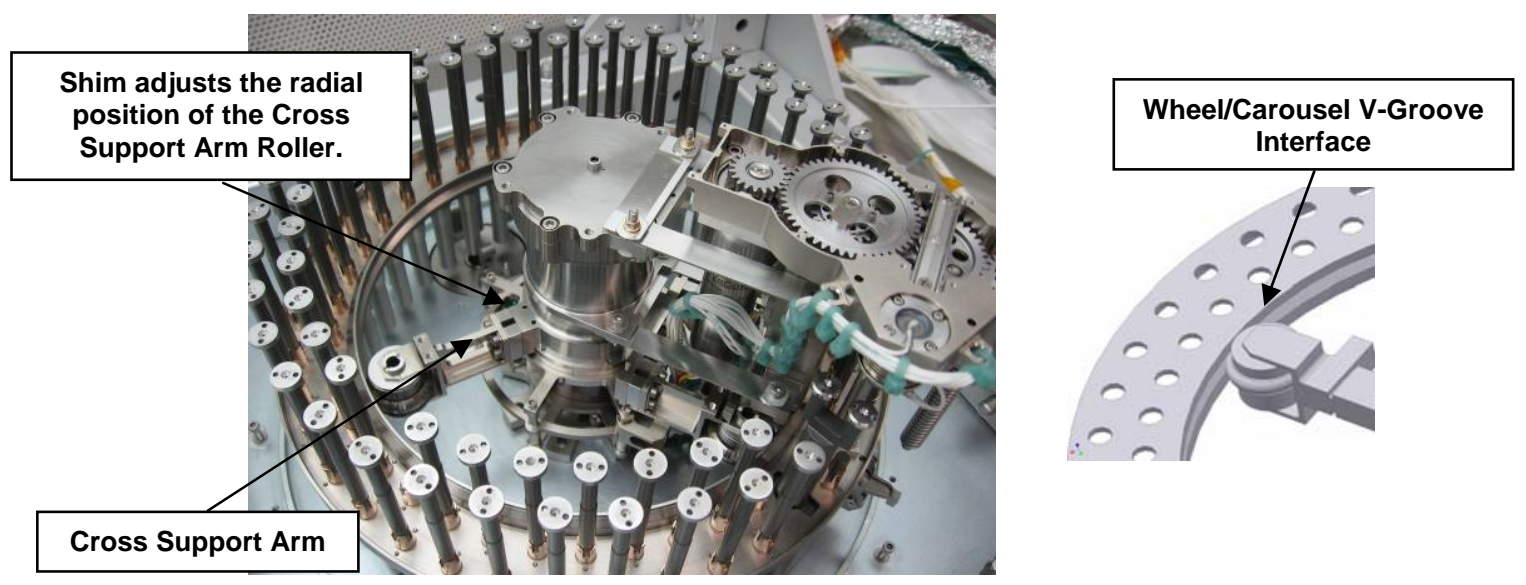

Figure 6 - Radial Adjustment Shim for Carousel Disk Concentricity

Using a single actuator to position both the Rotating Elevator Frame and the Sample Carousel Disk required more than $360^{\circ}$ of rotation of the center hub. A twist capsule carries power and signals to the elevator actuator and feedback switches on the Rotating Elevator Frame. To prevent damage to the twist capsule in the event of actuator run-off, hard stops were implemented in the Center Hub. To allow for greater than 1 revolution of travel, an intermediate hard-stop ring travels between the ground and the output of the center hub, Figure 7 . This design allows for $693^{\circ}$ of rotation, hard stop to hard stop.

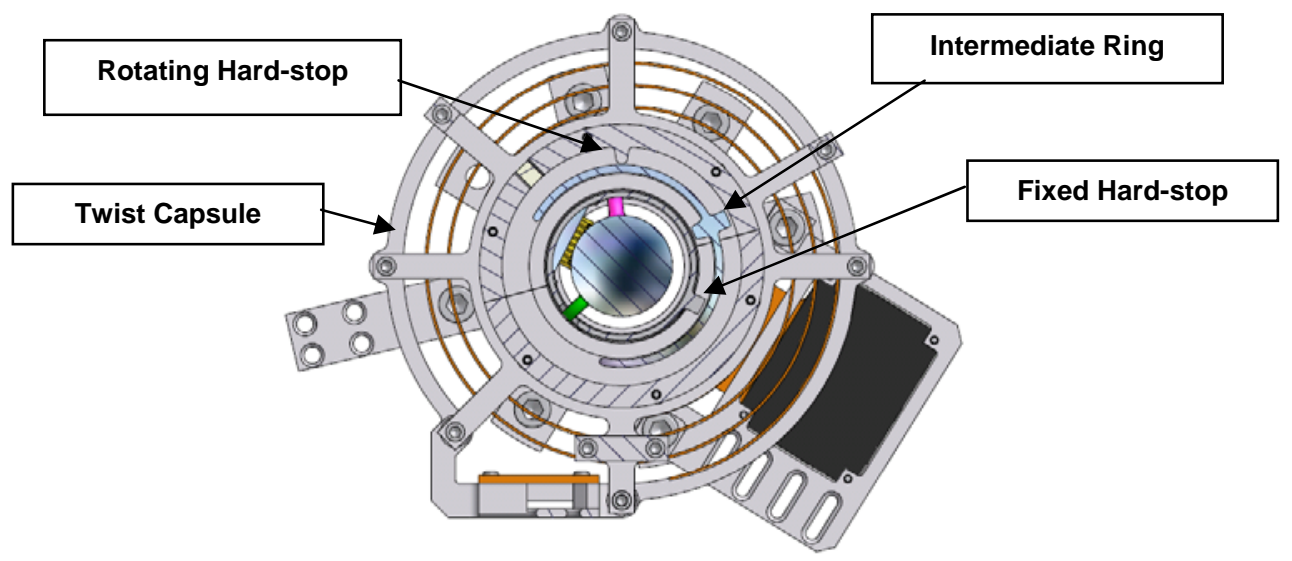

Figure 7 - Top Section View of Center Hub Twist Capsule and Hard-stops

Rotating Elevator Frame

The Rotating Elevator Frame consists of a linear actuator to raise and lower cups, a parallel spring flexure, and a launch lock for the elevator subassembly, Figure 8. The parallel spring flexure allows the 
Elevator launch lock cleats to de-mate when there is no load applied to the elevator lift via a sample cup. This system allows for a re-usable launch lock mechanism and a reduction in structural mass required to deliver a seal force to a sample cup or the contamination seal. The 1350 Newtons that the elevator delivers is all reacted locally, Figure 9 . The load path does not trace back through the center hub assembly, which would have required significantly more structural mass to accommodate.

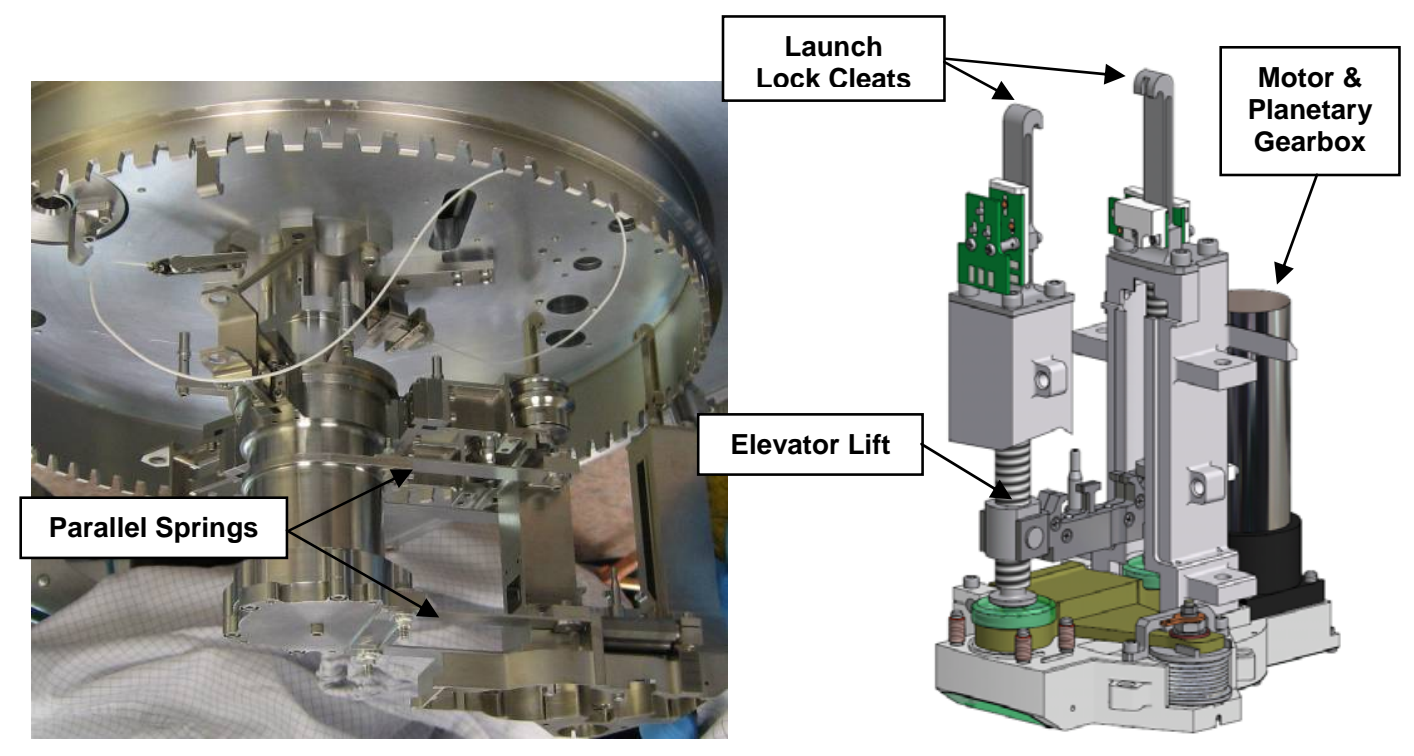

Figure 8 - Elevator Actuator \& Rotating Elevator Frame
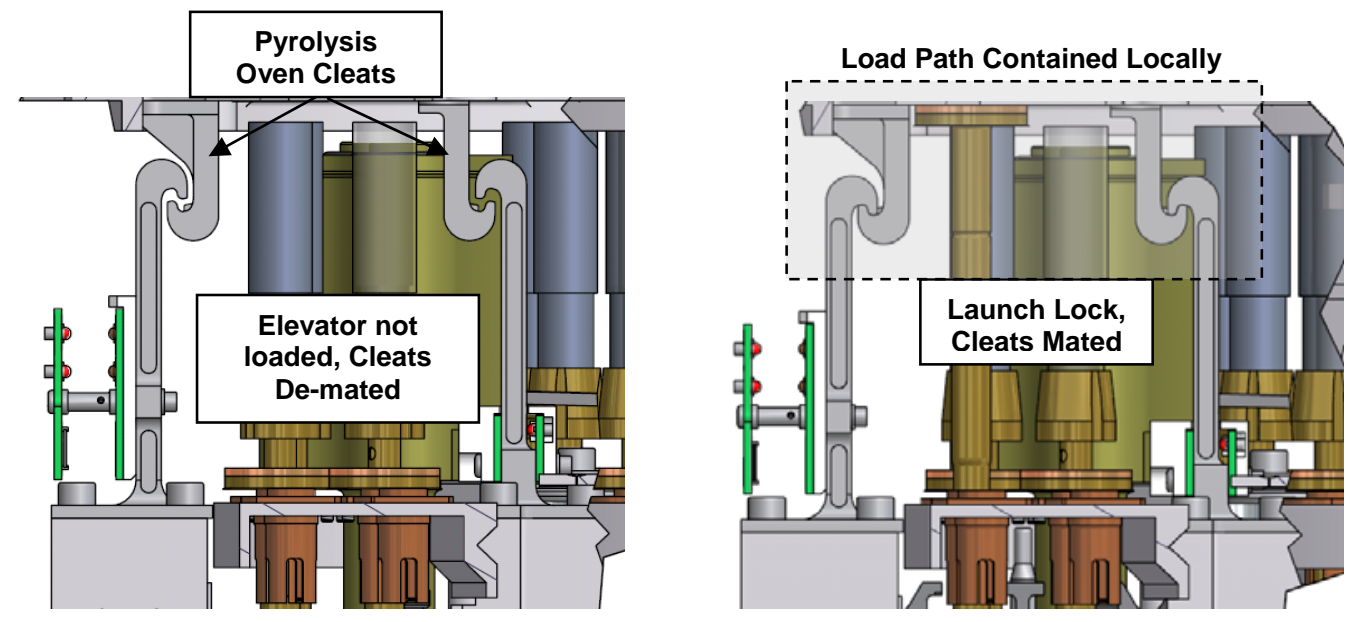

Figure 9 - Rotating Elevator Frame Launch/Seal Lock

\section{Elevator Actuator}

The linear actuator for the Elevator consists of a hall commutated brushless DC motor, 80:1 planetary gearbox, 2.4:1 spur gear arrangement, and a pair of 5/16-12 ACME lead screws, resulting in resolution of 1088 hall counts per millimeter. The two lead screws are mechanically coupled via Custom $455 \mathrm{H} 1000$ Stainless Steel spur gearing in the bottom of the Elevator Assembly. The two lead screws are Nitronic 60, $50 \%$ Cold Worked Stainless Steel and the ACME nuts are 15-5PH H1025 Stainless Steel. The ACME screw is non-backdriveable for coefficient of friction $>0.09$. A magnetic detent brake on the motor rotor is sufficient to hold the elevator preloaded in the event there is no friction in the leadscrew nut. The lead screws rotate in Nitronic 60, 20\% Cold Worked Stainless Steel journal bearings and 440C needle roller thrust bearings. The screws, nuts, bearings and gears are grease plated with Braycote 600 EF Micronic. Reservoirs in the journal bearings and the root of the ACME screws were also packed with the same grease. 


\section{Elevator Seal Force}

The elevator actuator is required to deliver $1350-\mathrm{N}$ force to a sample cup to an accuracy of $\pm 10 \%$ of the commanded force. The force delivered by the actuator must be commandable to allow for multiple uses of a single sample cup. An appropriately sized load cell could not be found that could withstand the temperature range $\left(-55\right.$ to $\left.110^{\circ} \mathrm{C}\right)$. A desire to reduce the burden on the control electronics and the added cost of development and qualification of a load cell led to an alternate solution custom tailored to the SMS design.

The load path for the seal force is transmitted through the lead screws and thrust bearings to a compliant flexure arm, Figure 10. The lead screws and lift assembly are allowed to translate along the axis of the applied load. A spherical shoulder on the bottom of each lead screw allows the flexure arm to rotate about its hinge axis without inducing moments in the spur gearing and the leadscrew/nut interface. Further, the lift assembly is pinned to each leadscrew nut to allow the lift to flex without inducing moments in the lead screws, thereby maximizing the efficiency of the screw/nut interface. At the end of the flexure arm is a spring-loaded electromechanical switch. With no load applied, the switch is an open circuit. Once a load is applied to the lift, the switch begins to close. Once the load reaches 450 Newtons, the switch closes. A short stroke wave spring provides the repeatable contact force for the switch assembly. Once the switch closes, the structure becomes a linear spring. The stiffness of the structure, in units of motor counts per Newton, is determined during mechanism calibration. Therefore, once the switch closes at a known force, all backlash in the load path and drivetrain has been eliminated. Commanding the motor to run a specific number of hall counts past the switch transition will result in the desired seal force applied to the sample cup. An integrated bellows assembly keeps the switch contact area free of particulate and Martian dust.

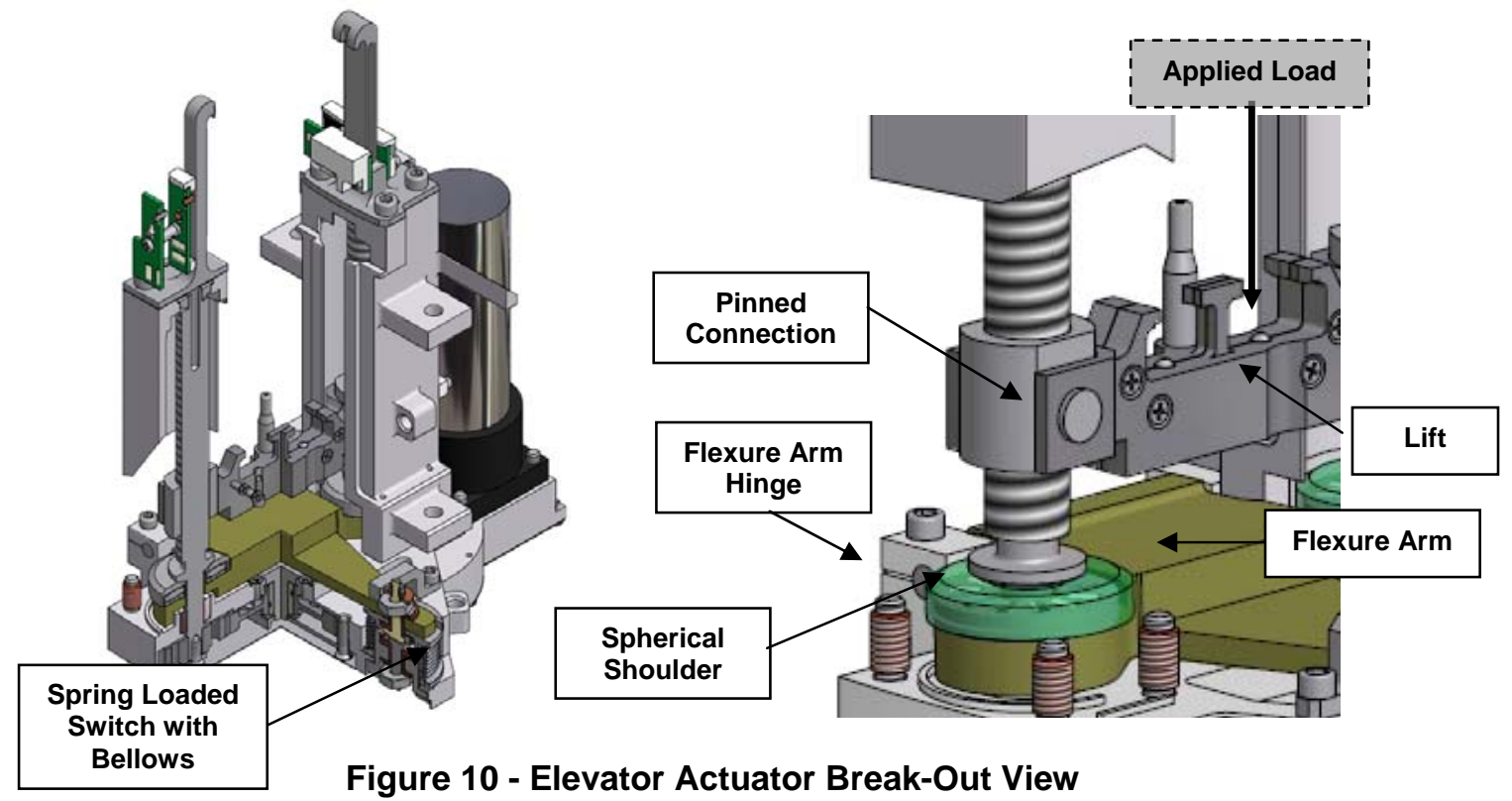

Incremental Encoder

Optical switches reside at the output of the Rotating Elevator Frame and consist of opposed LEDs and Phototransistors. A pair of LED/PT switches, together with the tooth profile on the Ground Ring act as a custom incremental encoder located at the output of the mechanism, Figure 11. The "region of alignment" indicates the REF is in a position where it is acceptable to actuate the toggle mechanism or raise and lower a cup. By integrating feedback to the output of the mechanism, motor hall counts can be compared to optical switch state transitions to detect faults by a prompt, safe, and recoverable means. Together with the hard-stops on the center hub, the SMS is capable of full recovery from an unexpected loss of power that would result in the loss of position information. The SMS may also operate should either the motor hall sensors or the optical switches fail. It is a redundant control scheme. 


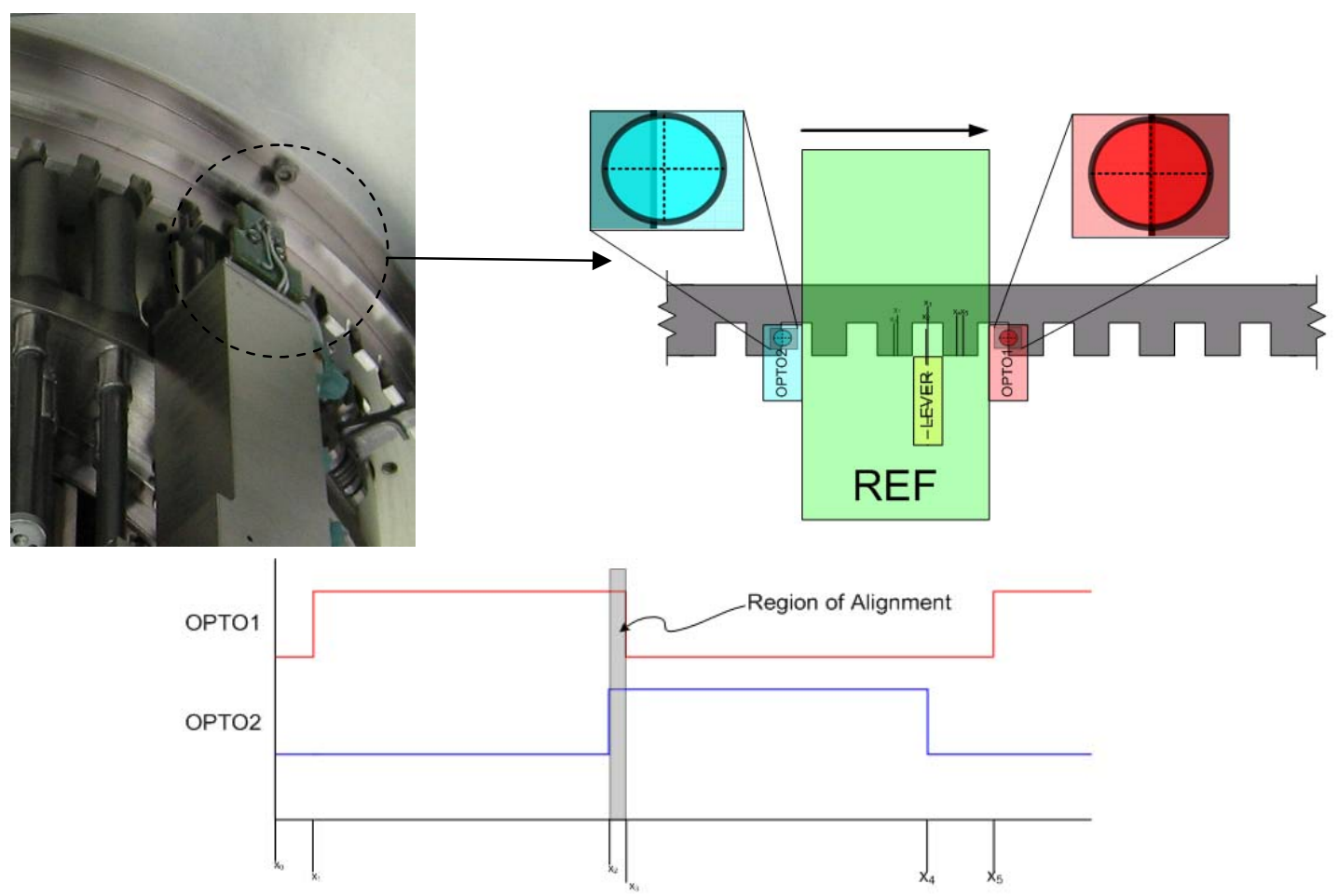

Figure 11 - REF Incremental Encoder

\section{Sample Carousel Disk}

The Sample Carousel Disk holds all 74 sample cups, the toggle mechanism, and the Vacuum Seal and excess sample container, Figure 12. There are 37 sample cups located on each row of the Sample Carousel Disk. The packing density of the Sample Carousel was maximized to provide as many clean cups as possible within the allotted volume. Each sample cup can interface with the puncture station, pyrolysis oven, and sample inlet on its respective row. Two rows of cups provides for redundancy in the event of a pyrolysis oven failure.

In the launch position, the vacuum seal is elevated at the solid sample inlet location. O-rings provide a vacuum tight seal to prevent the sample cups from being contaminated prior to arrival at Mars. Also integrated into the vacuum seal is a reservoir to collect excess sample flushed through the inlet tubes. When the vacuum seal is in place, the launch lock on the elevator assembly is active. Each sample cup is constrained to the Sample Carousel Disk by a cantilever spring detent release/restraint device. The sample cup restraint must hold the sample cups during launch and EDL activities and release when the cup is used for an experiment on Mars. 


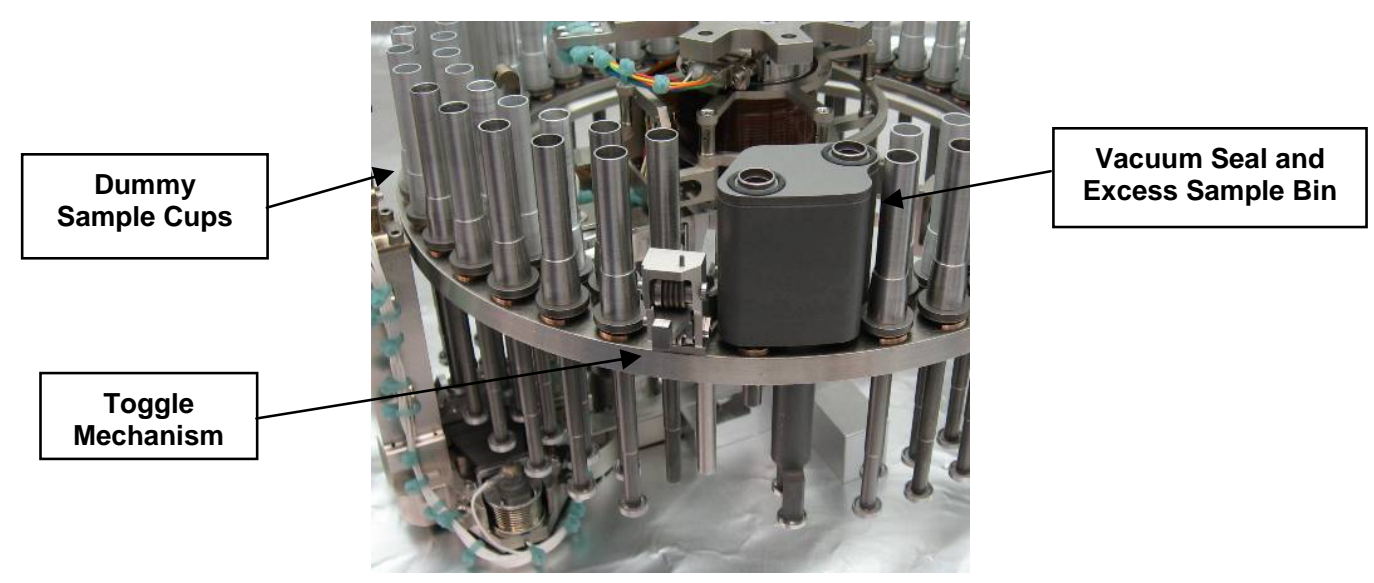

Figure 12 - Sample Carousel Disk

\section{Test Results and Lessons Learned}

The SMS Flight Model has recently completed its Flight Model Proto-flight test campaign. An Engineering Model was also subject to an extended proto-flight test program and $2 X$ life test. Several lessons learned are reported here as a result.

\section{Sample Cup Launch Restraint}

The launch restraint for the sample cups required a redesign during Engineering Model Testing. The initial design utilized a canted coil spring to restrain each cup during launch and EDL events. A canted coil spring is often used in spring-loaded shaft seals to provide low friction mitigation against dust and debris on rotating shafts. The canted coil springs can also be used as a detent device for a shaft sliding through a cylinder. The initial design utilized an Inconel X-750 canted coil spring housed in a Nitronic 60 Stainless Steel bushing, Figure 13. The sample cup stem is Titanium 6AL-4V plated with a Canadize ${ }^{\mathrm{TM}}$ coating (Polymer infused Titanium Oxide) from General Magnaplate Corporation. A groove on the Titanium Sample Cup Stem allowed the spring to constrict and consequently provide a restraint for the sample cup. When the sample cup is used, the Elevator actuator would apply an axial force to the sample cup stem and cause the canted coil spring to "dilate", thereby releasing the cup from its restraint.

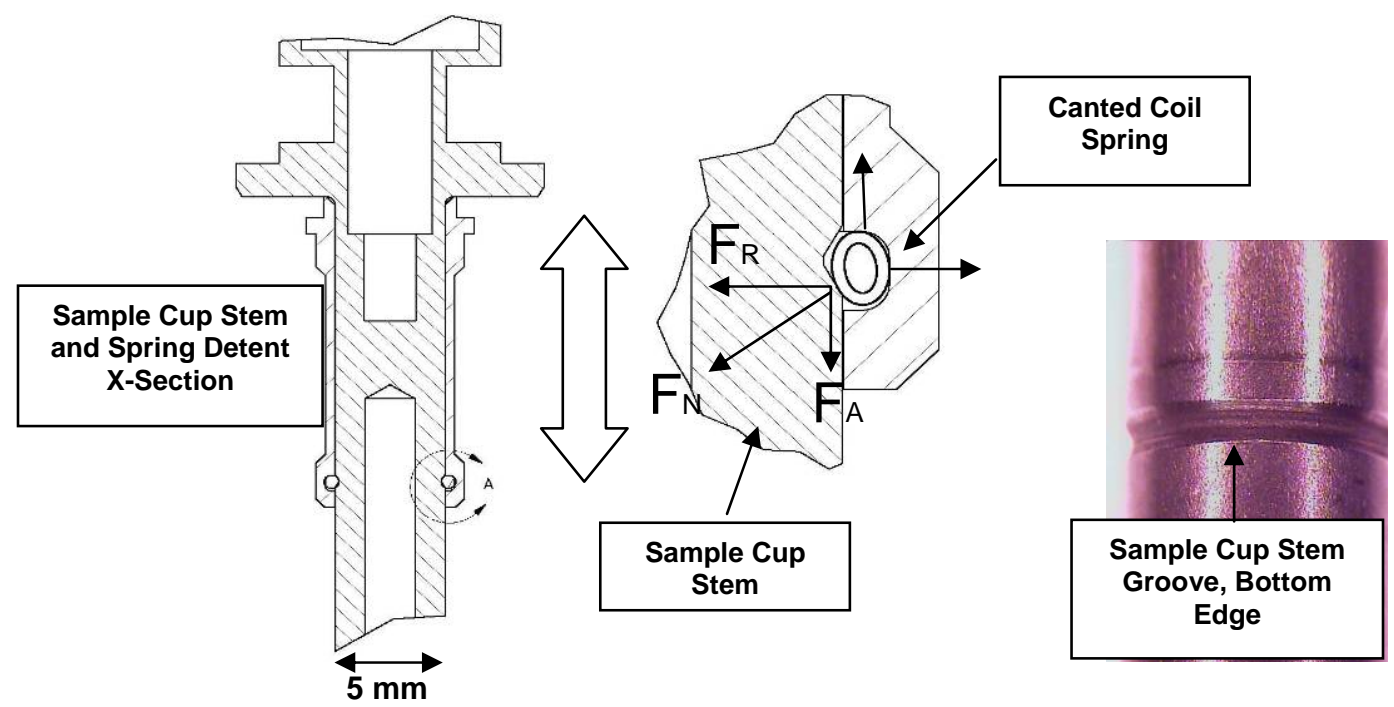



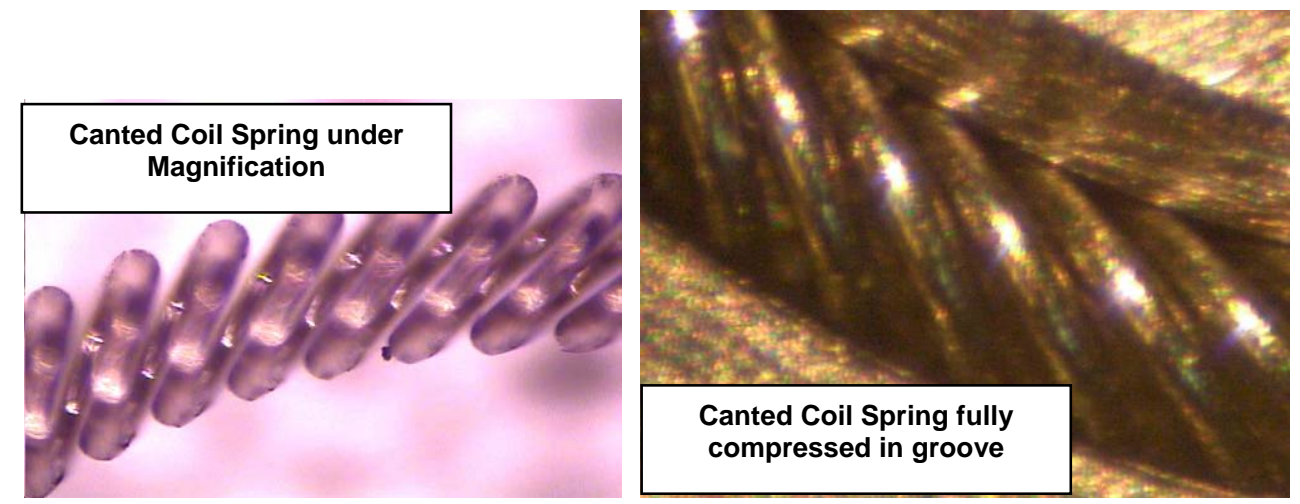

Figure 13 - Initial Sample Cup Restraint

There were several issues with this design. Initially the restraint force was not sufficient to hold the cups in place with sufficient margin during launch. The Sample Carousel Disk can see responses peaks as high as $130 \mathrm{Gs}$ during random vibe. To address this issue, the bushing groove diameter was reduced to radially compress the spring more, resulting in a higher detent force. Once all the parts were precision cleaned in ultrasonic cleaners through multiple baths (3 IPA, 1 Simple Green, 1 Acetone), the coefficient of friction between the spring and the sample cup stem increased. The resulting spring deflection was also getting close to the solid "height" of the spring. Thus, the behavior of the restraint/release device behaved differently in the clean room than in the pre-cleaned state. Subsequent testing on the Engineering Model in the clean room showed signs of erratic behavior. It proved to be very difficult to pair springs with sample cups. Eventually a point was reached where the Carousel was fully populated with cups exhibiting "acceptable" performance.

The Engineering Unit was then subject to a vacuum bake-out at $90^{\circ} \mathrm{C}$ for 24 hours. Following the bakeout, the cup stems exhibited stick-slip behavior, which was not entirely unexpected given the performance in clean room bench testing. Most of the cup stems would only release after a much higher load was applied by the Elevator actuator than was previously necessary. Some did not release at all. It was determined that the combination of increased compression on the canted coil spring, precision cleaned components lacking surface lubricants (particularly after the vacuum bake-out), and a sharp corner on the bottom edge of the sample cup groove (Figure 13) resulted in high contact stresses at the spring/stem interface which caused the intermittent and unreliable behavior of the restraint/release device. Some cup stems were then modified with a radius on the bottom edge of the groove in the sample cup stem. Preliminary testing showed promise that eliminating the sharp corner on the groove and thereby reducing the contact stress at the interface would allow the cup stem to release in a predictable and reliable manner.
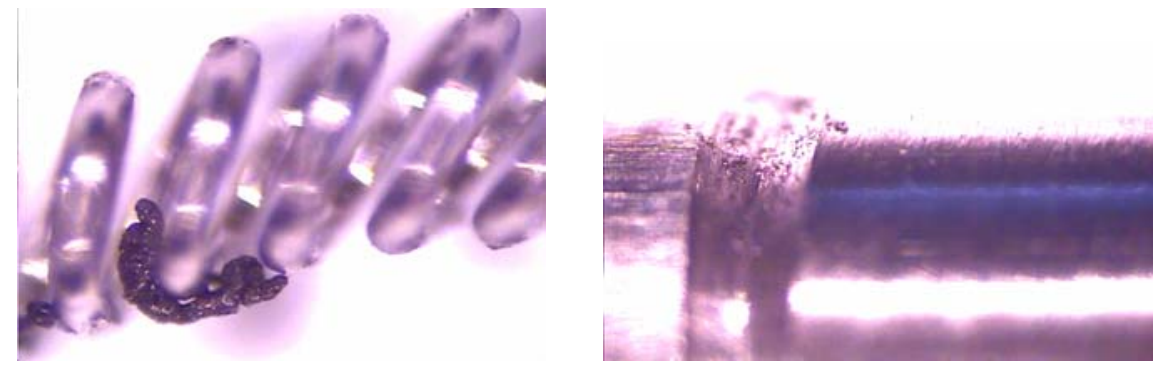

Figure 14 - Post Random Debris in Cup Stem Restraint Device

Six of the modified cup stems were then integrated into the sample carousel disk for vibration testing. The lateral $X$ and $Y$ tests were performed first, in which the in-axis was normal to the center axis of the sample cup. The Z-axis test aligned with the cup's major axis. On the full level random test, 5 of the 6 modified 
cup stems released from their restraint. None of the remaining 68-cup stems released during the test. However, in post-test performance testing, the unmodified (sharp groove corner) cup stems did not release. Inspection identified the cause as significant debris generation due to the random vibration environment. The debris lodged in between the coils of the spring and seized the release mechanism entirely, Figure 14.

The design that worked in T/V testing did not pass Random Vibe and vice versa. The proposed solution was to add another spring to the top end of the sample cup bushing in an attempt to stabilize the cup stem and prevent it from rattling and generating debris in vibration. The compression of the spring was also reduced to keep contact stresses as low as possible. Several variations utilizing two springs were tried. A component level random vibration test was then performed based on the response seen on the Carousel Disk during the system test. There were no candidate solutions based on the two-spring concept as a result of the test. The springs were not sufficient to prevent the sample cup stem from rattling in the bushing and generating significant debris.

The final design eliminated the canted coil spring entirely, Figure 15. The springs were replaced by integrated cantilevered beams that snap into the same groove in the sample cup stems that the spring used. The cantilever beam bushing is Brush Wellman Alloy 25 Copper Beryllium in the $\mathrm{TH}-04$ condition. The bushing was also electro-polished to reduce surface roughness and contact stresses. A much more repeatable and reliable restraint and release mechanism has been evident with this design. It does not allow the cup stem to rattle during random vibration and contact forces between the beams and the cup stem are easier to predict and control.
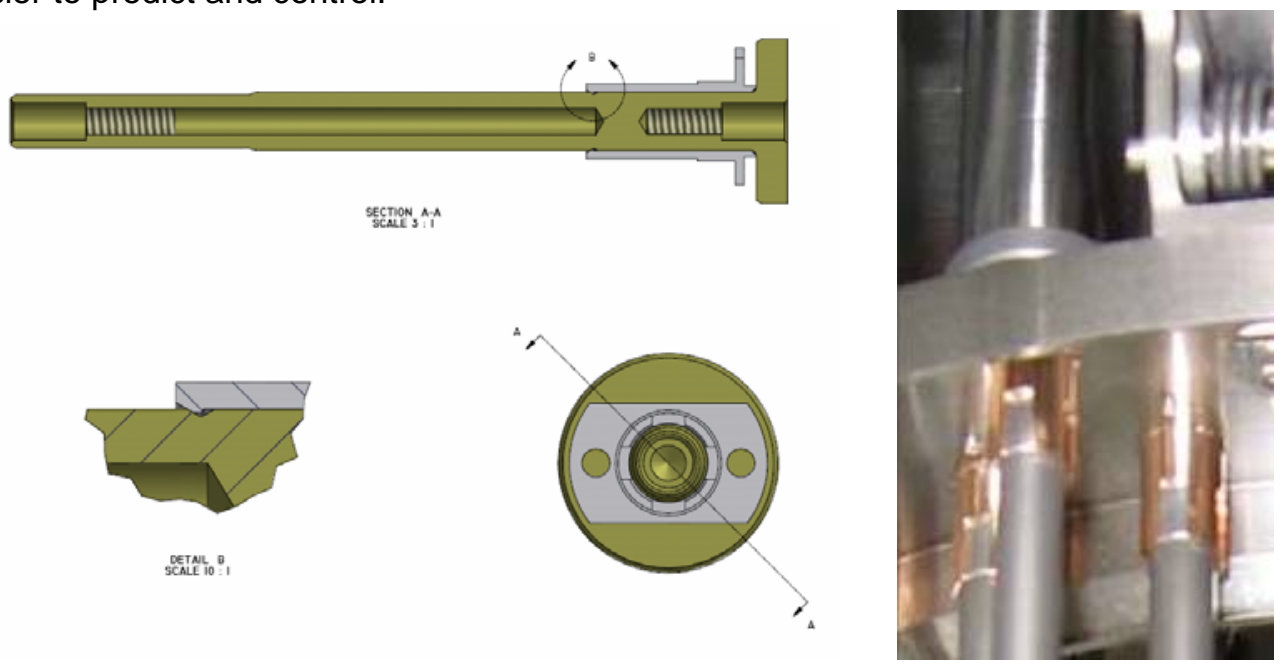

Figure 15 - Final Sample Cup Restraint Design

The major lessons learned from the sample cup restraint exercise are as follows.

1. Beware of lab test results that involve friction. Always precision clean parts to eliminate any oils or other lubricants that can collect in a lab environment. Precision cleaned parts behave differently than pre-cleaned parts.

2. Do not rely on components that are difficult to analyze and debug for a critical restraint application. The form factor of the canted coil spring and its release behavior made analytical predictions prone to large errors. The spring interaction with the cup stem was difficult to model and hidden from view.

3. If a canted coil spring is used to provide a detent for a component, ensure the vibration environment is well understood and excessive debris will not result. The canted coil spring can be a good solution for light detent applications if used in a conservative manner.

4. Be aware of the lap joint in the spring where the wire is welded. If not considered, the weld can also add to non-linearities/asymmetry at the interface between the two components. If high contact stresses exist, the weld may be problematic. 


\section{Contamination}

Another lesson learned in the development of the SMS pertains to contamination. When designing an instrument with extremely stringent outgassing and contamination requirements, work with all third party suppliers early and often on the contamination front. Expensive bake-outs resulted because assembled components such as motors were manufactured without additional contamination control guidelines in place. Once components are assembled, the vent path for all outgassing molecules becomes long and tortuous. The SMS motors required a 6-week bake-out before they were acceptable to use. Eliminating excessive polymeric harness staking and connector potting can also drastically reduce bake-out time and cost. Integrate metallic features in parts to tie harnesses down to rather than staking with polymerics. Avoid thick layers of polymerics. The thicker the layer, the longer it takes to diffuse and the longer the bake-outs become. The science return of the mission would have been compromised had the bake-outs not been sufficient.

\section{Optical Switches}

Optical switch thresholds will change with temperature. Be mindful when using optical switches to zero position counters in mechanisms. If position counters are reset with an optical switch transition, ensure the behavior of that switch has been characterized through its temperature range, particularly if a fine aperture is not implemented. Testing on the LED/PT switches used in the SMS demonstrated a shift with temperature in the threshold voltage of the optical switch, Figure 16. The SMS implemented a differential switch scheme with the custom incremental encoder that cancelled out the temperature shift. Optical switches on the elevator mechanism did not require fine positioning that counter resets would affect.

\section{Change in Transition Over Temperature}

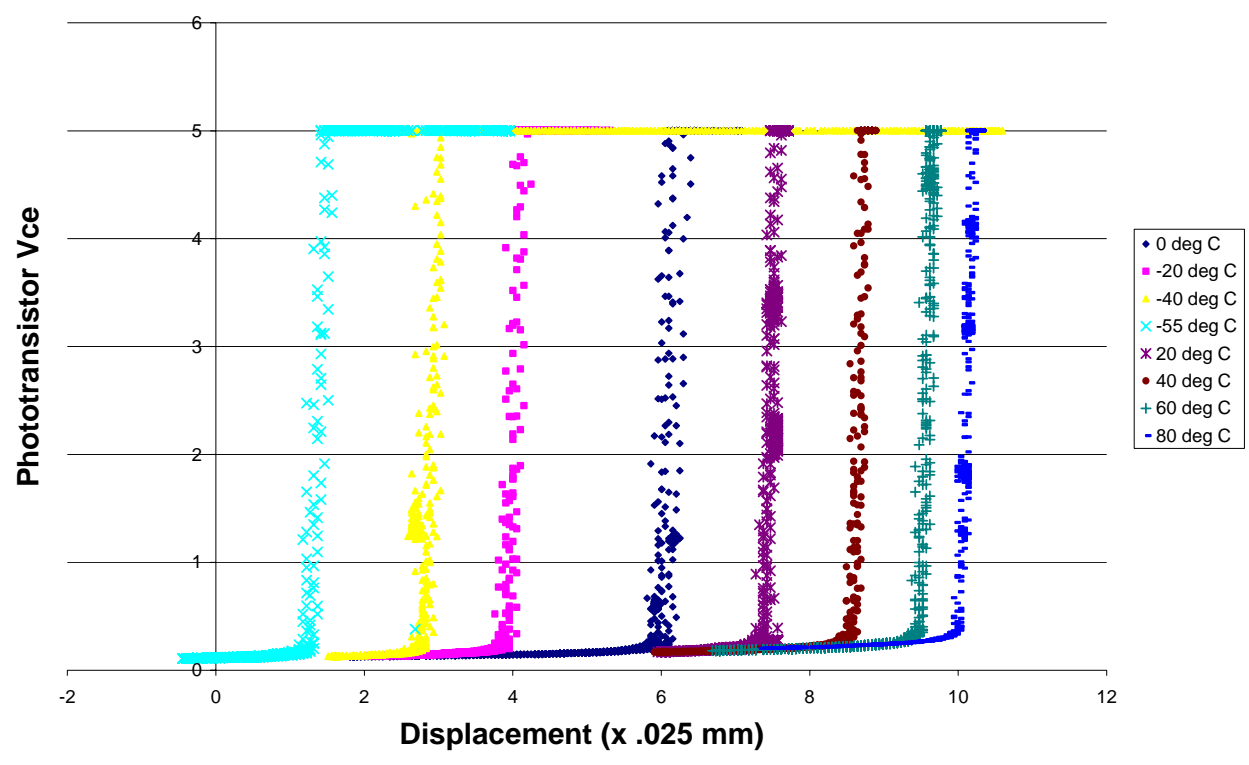

Figure 16 - Optical Switch Performance Vs Temperature

\section{Seal Force Feedback}

The performance of the Load Cell replacement in the Elevator Actuator mechanism was sufficient to satisfy SMS requirements. Paramount to the success of the design was a reliable switch closing force. So long as the force at which the switch closed remained consistent and was sufficient to remove any structural or electromechanical play in the load path and drive train, the stiffness of the structure can be utilized to apply a given force. Take care to tune the structural stiffness to the accuracy requirements and ensure the actuator feedback device has sufficient resolution to stay well within the required force accuracy, Figure 17. This is an effective method for applying a known force to a large number of distinct components of varying geometries. 


\section{Elevator Stiffness Calibration}

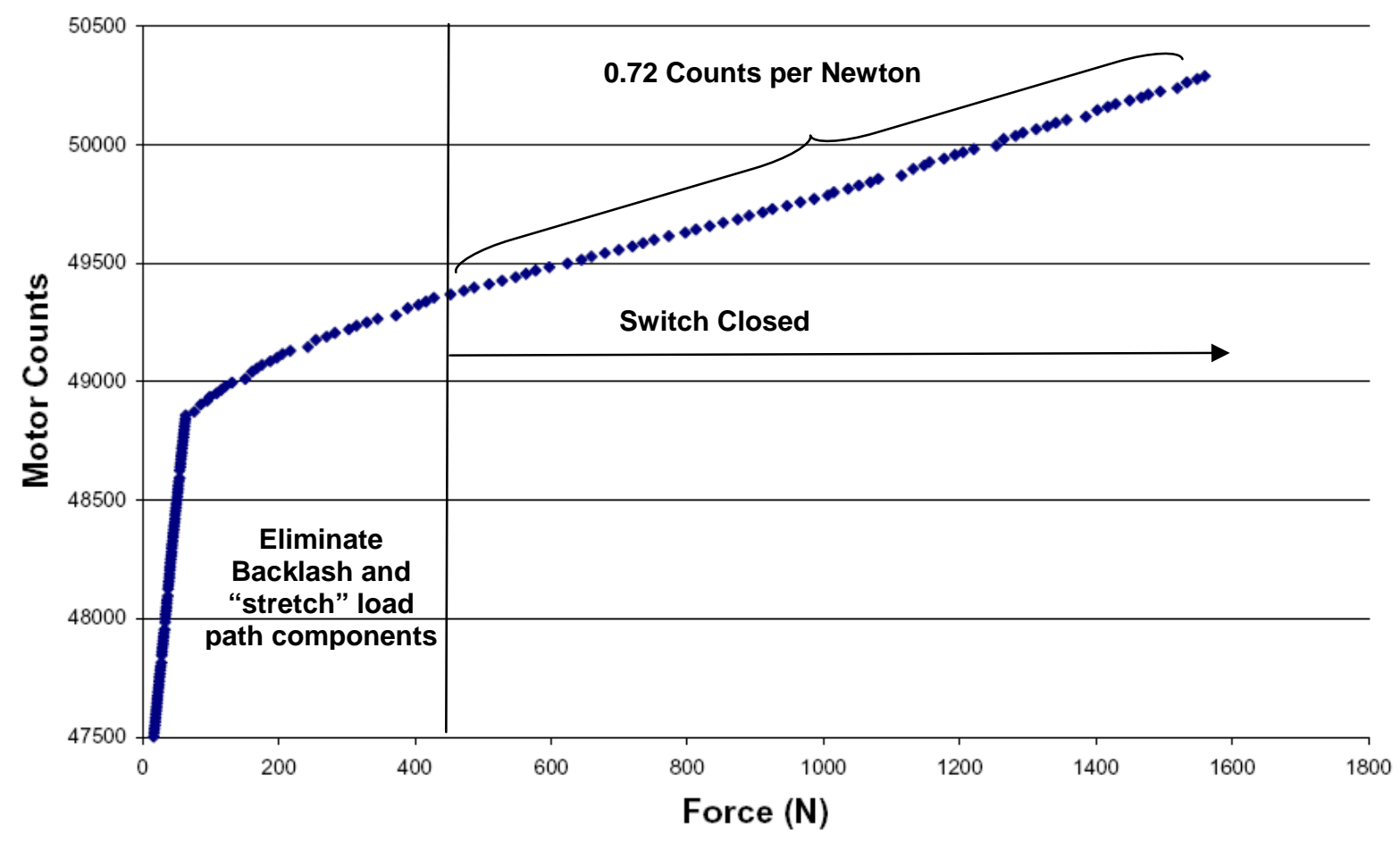

Figure 17 - Elevator Stiffness Calibration

General Test Data

Figure 18 plots the motor current versus time of the SMS during a complete Pyrolysis Experiment sequence. The sequence includes two sample cup seals, four Sample Carousel Disk re-orientations, and one vacuum seal/launch lock operation. Only the profile at $-55 \mathrm{C}$ is shown. The SMS motor currents were approximately $30 \%$ less at the maximum operating qualification temperature $\left(70^{\circ} \mathrm{C}\right)$. 


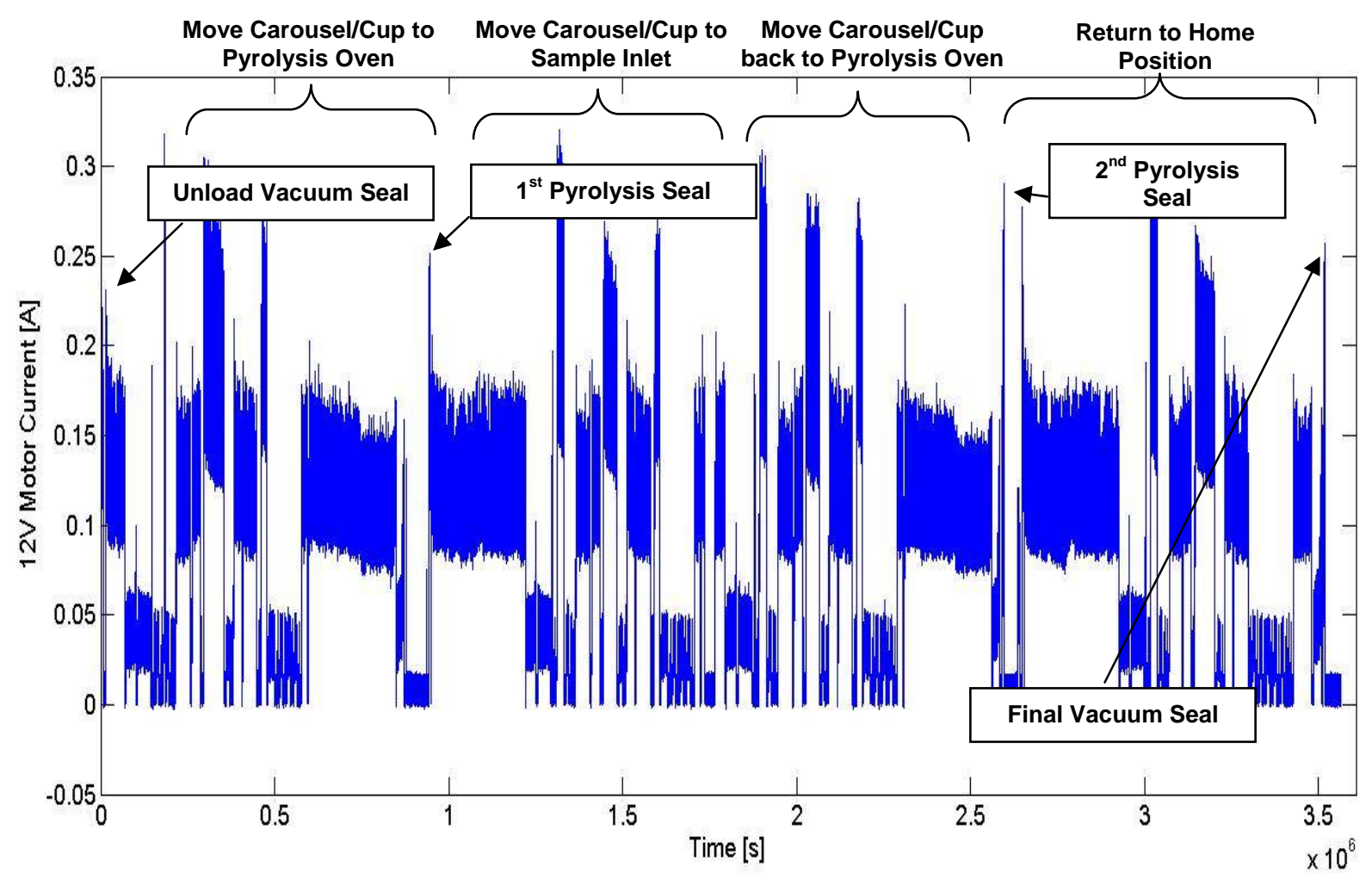

Figure 18 - Pyrolysis Experiment Current Profile

\section{Conclusions}

The Sample Manipulation System provided many challenges to the design team. Keys to designing mechanisms for very sensitive science experiments are to get involved with third party vendors as early as possible in the manufacturing stage. Assess the feasibility to bake-out sub-components prior to assembly. The problems encountered with using canted coil spring detents were a design oversight and should not be construed as a recommendation against using them for other applications. The debris generated inside the sample cup bushing demonstrated that another method for positive retention of the sample cup was necessary. The canted coil springs proved to be effective when used as a light detent device. Optical switches will drift with temperature. If a fine aperture is not implemented, the voltage threshold on the Phototransistor will occur at different degrees of LED obscuration. Lastly, the compliance of a structure can be an effective means of delivering a commandable force via an electromechanical actuator. All structural and drivetrain backlash must me eliminated prior to initiating the position move to a desired force. One means of doing so is implementing a repeatable spring-loaded switch to put the mechanism at a known point on the stiffness calibration curve.

\section{Acknowledgments}

The authors would like to thank all of the SMS program technicians, machinists, designers, engineers and managers at Honeybee Robotics and GSFC for their commitment and expertise. Specifically, the authors would like to thank Oren Sheinman and Rodger Farley of GSFC and Tom Myrick for their time and technical insights.

This work was performed under a contract with NASA Goddard Space Flight Center, Greenbelt, Maryland. References herein to any specific commercial product, process or service by trade name, trademark, manufacturer, or otherwise does not constitute or imply its endorsement by the United States Government or the Goddard Space Flight Center. 